\title{
CEUS: what is its role in abdominal aortic diseases?
}

\author{
Vito Cantisani $^{1}$, Emanuele David ${ }^{1,2}$, Liberatore Mauro' ${ }^{1}$ Ferdinando D’Ambrosio', \\ Dirk André Clevert ${ }^{3}$
}

${ }^{1}$ Department of Radiology, Anatomical-Patology and Oncology, Sapienza University of Rome, Rome, Italy, ${ }^{2}$ Department of Biomedical Sciences and Morphologic and Functional Imaging, Policlinico "G. Martino", University of Messina, Italy, ${ }^{3}$ Institut für Klinische Radiologie, Klinikum der Ludwigs-Maximilian-Universität München, Campus Großhadern, München, Deutschland.

In the last decade the sensitivity and specificity of contrast enhanced ultrasonography (CEUS) has greatly improved due to the development of more sophisticated ultrasonographic equipment, the introduction of secondgeneration contrast agents, and the development of dedicated software able to perform quantitative analysis [1]. Therefore, nowadays CEUS is established as a significant advancement in imaging and an effective technique with several clinical indications, especially for liver pathology as reported also in EFSUMB guidelines published in 2012 [2]. Apart from liver indications, CEUS has proved to be effective in several other fields [1,3] so that in 2011 EFSUMB reported their indications and recommendations for non-liver applications. These recommendations are based on comprehensive literature surveys including results from prospective clinical trials [4].

Among CEUS vascular indications related to abdominal aorta diseases were: aortic dissection, vascular stenosis, complications after vascular intervention, abdominal aortic aneurysm (AAA), inflammatory AAA, and endoleaks.

The most established recommendation was the detection, characterization, and follow up of endoleaks after AAA repair. In fact, CEUS is mainly employed in the assessment of abdominal vascular diseases to support the diagnosis of AAA and the evaluation of post operation complications.

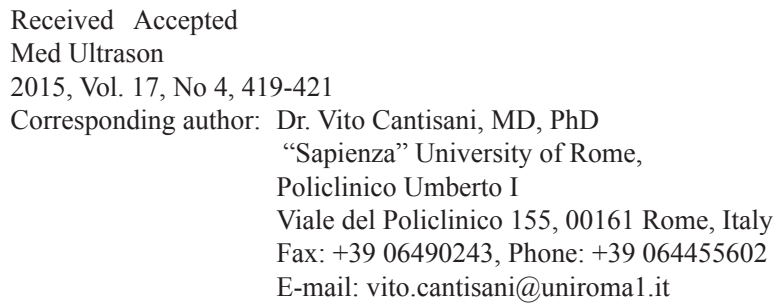

Nowadays the surgeons prefer to treat AAA by using EVAR (Endovascular Aneurysm Repair) and not by performing traditional surgery which is more invasive and aggressive and also incurs hospitalization and early mortality. The most frequent complication of EVAR is the endoleak, the incomplete exclusion of aneurismatic sac from the arterial circulation which can be classified in 5 types according to the Society for Vascular Surgery and the American Association for Vascular Surgery. In $10-45 \%$ of cases such complications can be associated with a dilation of the aneurysm sac and rupture $[5,6]$.

At the moment, CT angiography (CTA) is the reference diagnostic method, due to its wide availability, diagnostic value, acquisition speed, resolution, and uniformity of protocols [7]. However, it is an expensive method, uses ionizing radiation and potentially allergenic and nephrotoxic contrast agents.

As reported in literature, some valid alternatives to $\mathrm{CT}$ are CEUS and magnetic resonance angiography (MRA) $[8,9]$ but there is no consensus with regard to the optimal work-up with diagnostic imaging modalities in postEVAR surveillance [10]. CEUS could represent the ideal imaging modality to follow up EVAR.

The ideal imaging modality should be inexpensive, repeatable, safe, and accurate [11]. CEUS is cheaper than CTA, uses ultrasound, not ionizing radiation, and employs second generation contrast agents without nephrotoxic risks. It is safe and repeatable. Currently, in literature CEUS has given promising results for the identification of endoleaks and their correct classification [12]. Several authors have pointed out the usefulness of CEUS because it seems to identify and characterize endoleaks better than CTA, with an analysis of velocity and flow direction $[13,14]$. Moreover, CEUS enhancement quantification 
with Time-Intensity Curve (TIC) provides additional accuracy [15].

Conversely, CEUS still presents some clear limitations, especially if excess weight and meteorism are associated. Of particular note are also extensive wall calcification and subcutaneous emphysema after intervention, or limited examination windows [4].

Therefore, each imaging modality has advantages and disadvantages that should be considered when developing a surveillance program. CEUS offers several advantages and limitations compared to $\mathrm{CT}$, already mentioned. CTA is depicted by most as the pivotal imaging modality, while others consider CTA as unnecessary and expensive. MRA seems to offer better accuracy, but with higher costs and less availability [16].

Recently, a combined CEUS, color Doppler (CD) US, and CTA based protocol, that entails a base-line CTA at 3 months and 1 year post-EVAR, followed by periodic CDUS and use of 3D CEUS when a suspicious finding is detected, while MRA is performed in cases evidencing contraindications to CTA or in suspected endotension [10].

In a single case report, the authors reported an endovascular aortic repair in a patient with an asymptomatic infrarenal AAA and renal insufficiency. The precise placement of the stent-graft was performed with CEUS and intraprocedural angiographic fluoroscopy without the use of any nephrotoxic contrast media [17]. Intraoperative CEUS-assisted EVAR in patients with infrarenal AAA was reported to represent a new option for the visualization of aorto-iliac segments required as proximal or distal fixation zones and the identification of endoleaks, especially in those patients with contraindications for usage of iodine-containing contrast agents [18].

In a recently published review, Zimmerman et al summarized that CEUS is increasingly used and enables a quick, non-invasive follow-up examination for patient after EVAR. In addition, interventions such as therapy for endoleaks may be executed using ultrasound. Initial experience with CEUS-guided aortic stenting shows that the amount of contrast media as well as X-ray time may be reduced [19].

In conclusion, CEUS in the pre-, intra- and postEVAR surveillance is a safe and effective modality and in a well planned surveillance protocol it should be integrated into institutional protocols for EVAR surveillance in order to avoid the nephrotoxicity of contrast agents, the radiation and cost burden of repeated CTA-s in patients.

\section{References}

1. Cantisani V, Bertolotto M, Weskott HP, et al. Growing indications for CEUS: The kidney, testis, lymph nodes, thyroid, prostate, and small bowel. Eur J Radiol 2015; 84: 16751684.

2. Claudon M, Dietrich CF, Choi BI, et al; World Federation for Ultrasound in Medicine; European Federation of Societies for Ultrasound. Guidelines and good clinical practice recommendations for Contrast Enhanced Ultrasound (CEUS) in the liver - update 2012: A WFUMB-EFSUMB initiative in cooperation with representatives of AFSUMB, AIUM, ASUM, FLAUS and ICUS. Ultrasound Med Biol 2013; 39: 187-210.

3. Drudi FM, Giovagnorio F, Carbone A, et al. Transrectal colour Doppler contrast sonography in the diagnosis of local recurrence after radical prostatectomy--comparison with MRI. Ultraschall Med 2006; 27: 146-151.

4. Piscaglia F, Nolsøe C, Dietrich CF, et al. The EFSUMB Guidelines and Recommendations on the Clinical Practice of Contrast Enhanced Ultrasound (CEUS): update 2011 on non-hepatic applications. Ultraschall Med 2012; 33: 33-59.

5. Cao P, De Rango P, Verzini F, Parlani G. Endoleak after endovascular aortic repair: classification, diagnosis and management following endovascular thoracic and abdominal aortic repair. J Cardiovasc Surg (Torino) 2010; 51: 53-69.

6. Mehta M, Sternbach Y, Taggert JB, et al. Long-term outcomes of secondary procedures after endovascular aneurysm repair. J Vasc Surg 2010; 52: 1442-1449.

7. Thurnher S, Cejna M. Imaging of aortic stent-grafts and endoleaks. Radiol Clin North Am 2002; 40: 799-833.

8. Carrafiello G, Laganà D, Recaldini C, et al. Comparison of contrast-enhanced ultrasound and computed tomography in classifying endoleaks after endovascular treatment of abdominal aorta aneurysms: preliminary experience. Cardiovasc Intervent Radiol 2006; 29: 969-974.

9. Wieners G, Meyer F, Halloul Z, et al. Detection of type II endoleak after endovascular aortic repair: comparison between magnetic resonance angiography and blood-pool contrast agent and dual-phase computed tomography angiography. Cardiovasc Intervent Radiol 2010; 33: 1135-1142.

10. Cantisani V, Grazhdani H, Clevert DA, et al. EVAR: Benefits of CEUS for monitoring stent-graft status. Eur J Radiol 2015; 84: 1658-1665.

11. Corriere MA, Feurer ID, Becker SY, et al. Endoleak following endovascular abdominal aortic aneurysm repair: implications for duration of screening. Ann Surg 2004; 239: 800-805.

12. Cantisani V, Ricci P, Grazhdani H, et al. Prospective comparative analysis of colour-Doppler ultrasound, contrastenhanced ultrasound, computed tomography and magnetic resonance in detecting endoleak after endovascular abdominal aortic aneurysm repair. Eur J Vasc Endovasc Surg 2011; 41: 186-192.

13. Clevert DA, Minaifar N,Weckbach S, et al. Color duplex ultrasound and contrast-enhanced ultrasound in comparison to MS-CT in the detection of endoleak following endovascular aneurysm repair. Clin Hemorheol Microcirc 2008; 39 : 121-132.

14. Pfister K, Rennert J, Uller W, et al. Contrast harmonic imaging ultrasound and perfusion imaging for surveillance 
after endovascular abdominal aneurysm repair regarding detection and characterization of suspected endoleaks. Clin Hemorheol Microcirc 2009; 43: 119-128.

15. Jung EM, Rennert J, Fellner C, et al. Detection and characterization of endoleaks following endovascular treatment of abdominal aortic aneurysms using contrast harmonic imaging (CHI) with quantitative perfusion analysis (TIC) compared to CT angiography (CTA). Ultraschall Med 2010; 31: 564-570.

16. Habets J, Zandvoort HJ, Reitsma JB, et al. Magnetic resonance imaging is more sensitive than computed tomography angiography for the detection of endoleaks after endovascular abdominal aortic aneurysm repair: a systematic review. Eur J Endovasc Surg 2013; 45: 340-350.
17. Clevert DA, Kopp R. Contrast-enhanced ultrasound for endovascular grafting in infrarenal abdominal aortic aneurysm in a single patient with risk factors for the use of iodinated contrast. J Vasc Interv Radiol 2008; 19: 1241-1245.

18. Kopp R, Zürn W, Weidenhagen R, Meimarakis G, Clevert DA. First experience using intraoperative contrast-enhanced ultrasound during endovascular aneurysm repair for infrarenal aortic aneurysms. J Vasc Surg 2010; 51: 11031110 .

19. Zimmermann H, Reiser M, Meimarakis G, Paprottka P, Clevert DA. New Applications and Indications for Contrast-Enhanced Sonography in Endovascular Aortic Repair. Zentralbl Chir 2015; 140: 500-506. 